K. Miyake and N. Ormerod

Nagoya Math. J.

Vol. 95 (1984), 51-62

\title{
ABUNDANT CENTRAL EXTENSIONS OF NON-TRIVIAL GENERA
}

\author{
K. MIYAKE AND N. ORMEROD
}

\section{§1. Introduction}

Let $k$ be either a local or a global field, and $K$ be a finite Galois extension of $k$ with $\mathrm{g}=\mathrm{Gal}(K / k)$. Let $L$ be a Galois extension of $K$ which is also Galois over $k$. Such an extension is called central if $\operatorname{Gal}(L / K)$ lies inside the centre of $\operatorname{Gal}(L / k)$. Clearly $L$ is abelian over $K$. Next set $L^{*}=L \cap K \cdot k_{\mathrm{ab}}$ where $k_{\mathrm{ab}}$ is the maximal abelian extension of $k$ in its algebraic closure. This is the genus field of $L$ over $K / k$.

It is now well established that there is a connection between the theory of central extensions and the Schur multiplicator, $\mathrm{H}^{2}(\mathrm{~g}, \boldsymbol{Q} / Z)$, of g (see Fröhlich [1], and Miyake [4] for example). A direct approach to this connection is via a theorem of Tate and the Hochschild-Serre exact sequence.

TATE's TheOREm. Let $\Omega_{k}=\operatorname{Gal}(\bar{k} / k)$ where $\bar{k}$ is the algebraic closure of $k$. Then $\mathrm{H}^{2}\left(\Omega_{k}, \boldsymbol{Q} / Z\right)=0$. (See Serre [6, p. 227] in Fröhlich [2].)

Hochschild-Serre Exact Sequence. Let $N \triangleleft G$. Then there is an exact sequence

$$
\begin{aligned}
0 \longrightarrow \mathrm{H}^{1}\left(G / N, A^{N}\right) \longrightarrow \mathrm{H}^{1}(G, A) \longrightarrow \mathrm{H}^{1}(N, A)^{G / N} \\
\longrightarrow \mathrm{H}^{2}\left(G / N, A^{N}\right) \longrightarrow \mathrm{H}^{2}(G, A)
\end{aligned}
$$

for a $G$-module $A$. (See Hochschild-Serre [3].)

Now let $G=\Omega_{k}$, and $N=\Omega_{K}$, so that $\mathfrak{g}=G / N$. Then we have an exact sequence

$$
\begin{aligned}
0 \longrightarrow \mathrm{H}^{1}(\mathrm{~g}, \boldsymbol{Q} / Z) \longrightarrow \mathrm{H}^{1}\left(\Omega_{k}, \boldsymbol{Q} / \boldsymbol{Z}\right) \\
\longrightarrow \mathrm{H}^{1}\left(\Omega_{K}, \boldsymbol{Q} / Z\right)^{\mathfrak{s}} \longrightarrow \mathrm{H}^{2}(\mathfrak{g}, \boldsymbol{Q} / \boldsymbol{Z}) \longrightarrow 0 .
\end{aligned}
$$

A finite central extension $L$ is determined by a finite set $\left\{\chi_{1}, \cdots, \chi_{r}\right\}$ of

Received February 23, 1983. 
elements of $\mathrm{H}^{1}\left(\Omega_{K}, \boldsymbol{Q} / \boldsymbol{Z}\right)^{\mathrm{g}}$ such that $\bigcap_{i=1}^{r} \operatorname{ker}\left(\chi_{i}\right)=\Omega_{L}$. The kernel of the mapping

$$
t: \mathrm{H}^{1}\left(\Omega_{K}, \boldsymbol{Q} / Z\right)^{\mathfrak{g}} \longrightarrow \mathrm{H}^{2}(\mathrm{~g}, \boldsymbol{Q} / Z)
$$

is precisely the image of $\mathrm{H}^{1}\left(\Omega_{k}, \boldsymbol{Q} / Z\right)$ in $\mathrm{H}^{1}\left(\Omega_{K}, \boldsymbol{Q} / Z\right)$, i.e. the set of those characters of $\Omega_{K}$ which determine abelian extensions of $K$ lying in $K \cdot k_{\mathrm{ab}}$. From this discussion, we see that $\operatorname{Gal}\left(L / L^{*}\right)$ is isomorphic to the dual of the image of $\left\langle\chi_{1}, \cdots, \chi_{r}\right\rangle$ in $\mathrm{H}^{2}(\mathrm{~g}, \boldsymbol{Q} / Z)$.

Moreover since $\left|\mathrm{H}^{2}(\mathrm{~g}, \boldsymbol{Q} / Z)\right|$ is finite and the transgression mapping $t$ above is surjective, we easily see that there exists a finite central extension, $L$, such that $\mathrm{Gal}\left(L / L^{*}\right)$ is isomorphic to the dual group, $\subseteq(K / k)$, of $\mathrm{H}^{2}(\mathfrak{g}, \boldsymbol{Q} / Z)$. Such extensions are called abundant central extensions of $K / k$. In Miyake [4] the question was asked whether there exists an abundant central extension $L$ such that $L^{*}=K$. This question was further investigated in [5]. Here we shall prove by a careful analysis of the local cases of characteristic 0 that this is not always possible. This will allow us to construct examples of global extensions of algebraic number fields where it is impossible to construct abundant central extensions with the trivial genus fields.

Note that the following definition is natural since $\widetilde{S}(K / k)$ is abelian:

Definition. For a prime $p$, we say that $L$ is an abundant central $p$-extension of $K / k$ if

(i ) $L / K$ is a central $p$-extension, and

(ii) $\operatorname{Gal}\left(L / L^{*}\right)$ is isomorphic to $\mathfrak{S}^{(p)}(K / k)$ where $\mathfrak{S}^{(p)}(K / k)$ is the $p$-primary component of $\subseteq(K / k)$.

The following result is then typical of what we will prove.

TheOREM. Let $\nu$ be the discrete valuation of the local field $k$. If $\nu(p)=0$, then there is an abundant central p-extension $L$ of $K / k$ such that $L / K$ is cyclic and that

$$
\left[L^{*}: K\right]=\left|\mathrm{N}_{K / k}\left(\mu_{p^{\infty}} \cap K^{\times}\right)\right| .
$$

Furthermore, for any abundant central p-extension $M$ of $K / k$, we have

$$
\left[M^{*}: K\right] \geq\left|\mathrm{N}_{K / k}\left(\mu_{p^{\infty}} \cap K^{\times}\right)\right|
$$

unless $\mathfrak{\varsigma}^{(p)}(K / k)=\{1\}$.

Here $\mu_{p^{\infty}}$ is the group of $p$-power roots of 1 in $\vec{k}$. 
This work was done while the first named author was a Visiting Member of the School of Mathematics, the University of New South Wales. He would like to express his heartfelt thanks to the University for giving him the opportunity to work there, and to the members of the staff of the School for their hospitality he had recieved during his stay.

\section{§2. Local Cases I}

In this section and in the next we shall take our fields $k, K$ etc. to be completions of algebraic number fields at non-archimedian prime places. Our starting point is the following observation.

Proposition 1. Let $K$ be a Galois extension of $k$ with the Galois group g. Then $\subseteq(K / k)$ is isomorphic to $\mathrm{N}_{K / k}^{-1}(1) / K^{\Delta_{3}}$ where $\mathrm{N}_{K / k}: K^{\times} \rightarrow k^{\times}$ is the norm mapping, and $K^{\Delta_{\mathfrak{g}}}=\left\langle x^{1-\sigma} \mid x \in K^{\times}, \sigma \in \mathrm{g}\right\rangle$.

Proof. Dualise the exact sequence $(*)$ above and apply the Artin map of class field theory. (Cf. Miyake [4].)

Thus we may study central extensions by a careful analysis of the groups $K^{\times}, \mathrm{N}_{K / k}^{-1}(1)$ and $K^{\lrcorner_{s}}$.

For a profinite abelian group $A$, we denote the $p$-primary part of $A$ by $A^{(p)}$.

Let $V$ be an open subgroup of $K^{\times}$which determines an abelian extension $L$ of $K$ via class field theory. Then it is clear that $L$ will be an abundant central $p$-extension if and only if $V$ satisfies the following three conditions:

(i) $V \supset K^{\Delta_{9}}$;

(ii) $V \cap \mathrm{N}_{K / k}^{-1}(1)^{(p)}=\left(K^{\lrcorner_{s}}\right)^{(p)}$;

(iii) $\left[K^{\times}: V\right]=p^{m}$ for some $m \geq 0$.

Note that (i) implies $V^{o}=V$ for every $\sigma \in \mathrm{g}$. Therefore $L$ is certainly a Galois extension of $k$.

Given the above, the genus field $L^{*}$ of $L$ over $K / k$ has the degree over $K$ given by

$$
\left[L^{*}: K\right]=\left[K^{\times}: V \cdot \mathrm{N}_{K / k}^{-1}(1)^{(p)}\right] .
$$

Let $\nu$ be the valuation of the field $k$. Our analysis will vary according to $\nu(p)=0$ or $\nu(p)>0$.

Throughout we will write $\mu_{p}(K)$ for $\mu_{p^{\infty}} \cap K^{\times}$etc. 
Case 1: $\nu(p)=0$.

THEOREM 1. If $\nu(p)=0$ and $\mathfrak{S}^{(p)}(K / k) \neq\{1\}$, then there is an abundant central p-extension $L$ of $K$ over $k$ such that $L / K$ is cyclic and that

$$
\left[L^{*}: K\right]=\left|\mathrm{N}_{K / k}\left(\mu_{p}(K)\right)\right| \text {. }
$$

Furthermore for any abundant central p-extension $M$ of $K / k$, we have

$$
\left[M^{*}: K\right] \geq\left|\mathrm{N}_{K / k}\left(\mu_{p}(K)\right)\right| \text {. }
$$

Proof. We have a decomposition

$$
K^{\times}=\langle\pi\rangle \times \mu_{p}(K) \times U
$$

where $\pi$ is a prime element of $K$ and $U$ is a $p$-divisible subgroup of the unit group $U_{K}$ of $K$. Thus we have

$$
K^{\Delta_{\mathfrak{g}}}=\left\langle\pi^{1-\sigma} \mid \sigma \in g\right\rangle \cdot \mu_{p}(K)^{\Delta_{\mathfrak{g}}} \cdot U^{\Delta_{\mathfrak{g}}} .
$$

Since $\mu_{p}(K)$ and $U$ are g-stable, we have $\mu_{p}(K)^{\Delta_{\mathfrak{g}}} \subset \mu_{p}(K)$ and $U^{\Delta_{\mathfrak{g}}} \subset U$.

LEMMA 1. $\left(K^{\Delta_{g}}\right)^{(p)}=\mu_{p}(K) \cap K^{\Delta ?}$.

Proof. Let $e$ be the exponent of $U \cap N_{K / k}^{-1}(1) / U^{\Delta_{9}}$. Then $(e, p)=1$ because $U$ is $p$-divisible. Let $x$ be an element of $\left\langle\pi^{1-\sigma} \mid \sigma \in \mathfrak{g}\right\rangle$, and take $y \in\left(K^{\Delta_{g}}\right)^{(p)}$ and $z \in K^{\Delta_{\mathfrak{g}}} \cap U$ so that $x=y \cdot z$. Then $y^{e}=x^{e} \cdot z^{-e} \in\left\langle\pi^{1-\sigma}\right\rangle \cdot U^{\Delta_{\mathfrak{g}}}$ $\subset K^{\Delta_{8}}$. Since $y \in\left(K^{\Delta_{8}}\right)^{(p)} \subset \mu_{p}(K)$ and $(e, p)=1$, we have $y \in \mu_{p}(K) \cap K^{\Delta_{8}}$. This shows that $\left(K^{\Delta_{g}}\right)^{(p)} \subset \mu_{p}(K) \cap K^{\Delta_{g}}$. Since the converse inclusion is obvious, the lemma is proved.

Now, it is clear that

$$
\mathrm{N}_{K / k}^{-1}(1)^{(p)}=\left\{\zeta \in \mu_{p}(K) \mid \mathrm{N}_{K / k}(\zeta)=1\right\} .
$$

Thus provided $\varsigma^{(p)}(K / k) \cong \mathrm{N}_{K / k}^{-1}(1)^{(p)} /\left(K^{\iota_{g}}\right)^{(p)} \neq\{1\}$, then

$$
\left(U_{K} / K^{\Delta_{g}}\right)^{(p)}=\mu_{p}(K) / \mu_{p}(K) \cap K^{\Delta_{g}}
$$

does not split over $\mathrm{N}_{K / k}^{-1}(1)^{(p)} /\left(K^{\iota_{s}}\right)^{(p)}$ unless $\mu_{p}(K)=\mathrm{N}_{K / k}^{-1}(1)^{(p)}$ since $\mu_{p}(K)$ is cyclic. Hence we cannot find an open group $V$ which satisfies the conditions (i) $\sim$ (iii) above with

$$
\left[K^{\times}: V \cdot \mathrm{N}_{K / k}^{-1}(1)^{(p)}\right]<\left|\mu_{p}(K) / \mathrm{N}_{K / k}^{-1}(1)^{(p)}\right| .
$$

If we take

$$
V=\langle\pi\rangle \times\left(\mu_{p}(K) \cap K^{\lrcorner_{8}}\right) \times U
$$


then (i) (iii) are certainly satisfied, and we have

$$
\left[K^{\times}: V \cdot \mathrm{N}_{K / k}^{-1}(1)^{(p)}\right]=\left|\mu_{p}(K) / \mathrm{N}_{K / k}^{-1}(1)^{(p)}\right|=\left|\mathrm{N}_{K / k}\left(\mu_{p}(K)\right)\right| .
$$

Given our remarks above this proves the theorem.

Corollary. Suppose that $\nu(p)=0$ and $\widetilde{\varsigma}^{(p)}(K / k) \neq\{1\}$. Then there exists an abundant central p-extension of $K / k$ with the trivial genus field if and only if $\mathrm{N}_{K / k}\left(\mu_{p}(K)\right)=1$.

This follows from Theorem 1 at once.

Given these results it is not difficult to construct an example of an extension $K / k$ any abundant central extension of which has non-trivial genus.

Example 1. Let $\zeta_{m}$ denote a primitive $p^{m}$-th root of 1 chosen so that $\zeta_{m}^{p}=\zeta_{m-1}$. Suppose that $\zeta_{m} \in k$ but $\zeta_{m+1} \notin k$ for some $m>1$. Now let

$$
K=k\left(\sqrt[p]{\pi_{0}}, \zeta_{m+1}\right)
$$

where $\pi_{0}$ is a prime element of $k$. Then $\mathrm{g}=\mathrm{Gal}(K / k)$ is abelian of type $(p, p)$, which implies that $\mathrm{H}^{2}(\mathfrak{g}, \boldsymbol{Q} / Z)$ is cyclic of order $p$. Thus the conditions of the theorem are fulfilled, and it is easy to see that

$$
\left|\mathrm{N}_{K / k}\left(\mu_{p}(K)\right)\right|=\left|\mathrm{N}_{K / k}\left(\left\langle\zeta_{m+1}\right\rangle\right)\right|=\left|\left\langle\zeta_{m-1}\right\rangle\right|=p^{m-1} \geq p .
$$

Therefore for any abundant central extension $L$ of $K / k$ we have $\left[L^{*}: K\right] \geq p$.

\section{$\S 3 . \quad L o c a l$ Cases II}

Case 2: $\nu(p)>0$.

In this case $k$ and $K$ are finite algebraic extensions of the $p$-adic rational number field $\boldsymbol{Q}_{p}$.

Theorem 2. There exists an abundant central p-extension $L$ of $K / k$ such that $L^{*} / K$ is cyclic, and that

$$
\left[L^{*}: K\right] \leq\left|\mu_{p}(k) \cap \mathrm{N}_{K / k}\left(K^{\times}\right)\right| \text {. }
$$

Proof. Let $U_{k}$ and $U_{K}$ be the unit groups of $k$ and $K$ respectively. Then as a $Z_{p}$-module, $U_{k}^{(p)}$ is a direct product of the finite cyclic group $\mu_{p}(k)$ and a free $\boldsymbol{Z}_{p}$-module of $\operatorname{rank}\left[k: \boldsymbol{Q}_{p}\right]$. Put $d=\left[k: \boldsymbol{Q}_{p}\right]$. Since $\mathrm{N}_{K / k}\left(U_{K}^{(p)}\right)$ is a $Z_{p}$-submodule of $U_{k}^{(p)}$ of finite index, it is a direct product of $\mu_{p}(k) \cap \mathrm{N}_{K / k}\left(K^{\times}\right)$and a free $Z_{p}$-module of rank $d$. Choose $u_{1}, \cdots, u_{d} \in U_{K}^{(p)}$ so that $\mathrm{N}_{K / k}\left(u_{1}\right), \cdots, \mathrm{N}_{K / k}\left(u_{d}\right)$ generate over $Z_{p}$ a maximal free $Z_{p}$-submodule 
of $\mathrm{N}_{K / k}\left(U_{K}^{(p)}\right)$. Then the submodule $\left\langle u_{1}, \cdots, u_{d}\right\rangle_{Z_{p}}$ of $U_{K}^{(p)}$ generated by $u_{1}, \cdots, u_{d}$ over $Z_{p}$ is a free $Z_{p}$-module of rank $d$. Put

$$
X=\mathrm{N}_{K / k}^{-1}\left(\mu_{p}(k)\right)^{(p)}=\left\{x \in K^{\times} \mid x^{q} \in \mathrm{N}_{K / k}^{-1}(1)^{(p)} \text { for some } q=p^{m}\right\} .
$$

LEMMA 2. $U_{K}^{(p)}=X \times\left\langle u_{1}, \cdots, u_{d}\right\rangle_{z_{p}}$ (direct product).

Proof. The group on the right hand side is a subgroup of $U_{K}^{(p)}$, and mapped onto $\mathrm{N}_{K / k}\left(U_{K}^{(p)}\right)$. Since $X$ contains $\mathrm{N}_{K / k}^{-1}(1)^{(p)}=\mathrm{N}_{K / k}^{-1}(1) \cap U_{K}^{(p)}$, we have $U_{K}^{(p)}=X \cdot\left\langle u_{1}, \cdots, u_{d}\right\rangle_{z_{p}}$. Let $y \in X \cap\left\langle u_{1}, \cdots, u_{d}\right\rangle_{z_{p}}$. Then $\mathrm{N}_{K / k}(y)$ is a torsion element of $\left\langle\mathrm{N}_{K / k}\left(u_{1}\right), \cdots, \mathrm{N}_{K / k}\left(u_{d}\right)\right\rangle_{z_{p}}$. Since $\mathrm{N}_{K / k}\left(u_{1}\right), \cdots, \mathrm{N}_{K / k}\left(u_{d}\right)$ are free over $Z_{p}$, the element $y$ of $\left\langle u_{1}, \cdots, u_{d}\right\rangle_{Z_{p}}$ should be equal to 1 .

Q.E.D.

LEMma 3. $X \cap\left\{\left(K^{\Delta_{3}}\right)^{(p)} \cdot\left\langle u_{1}, \cdots, u_{d}\right\rangle_{z_{p}}\right\}=\left(K^{\Delta_{s}}\right)^{(p)}$.

This is clear by Lemma 2 because $X$ contains $\left(K^{\Delta_{g}}\right)^{(p)}$.

Lemma 4. For $x \in U_{K}^{(p)}, X=\langle x\rangle \cdot \mathrm{N}_{K / k}^{-1}(1)^{(p)}$ if and only if $\left\langle\mathrm{N}_{K / k}(x)\right\rangle=$ $\mu_{p}(k) \cap \mathrm{N}_{K / k}\left(K^{\times}\right)$.

Proof. It is obvious that $\mu_{p}(k) \cap \mathrm{N}_{K / k}\left(K^{\times}\right)=\mu_{p}(k) \cap \mathrm{N}_{K / k}\left(U_{K}^{(p)}\right)=\mathrm{N}_{K / k}(X)$. Since $\mathrm{N}_{K / k}(X)$ is cyclic, and isomorphic to $X / \mathrm{N}_{K / k}^{-1}(1)^{(p)}$, the lemma is clear.

Returning to the proof of Theorem 2, put

$$
V=\langle\pi\rangle \times \mu_{p}^{\prime}(K) \times\left(K^{\Delta_{s}}\right)^{(p)} \times\left\langle u_{1}, \cdots, u_{d}\right\rangle_{Z_{p}}
$$

where $\mu_{p}^{\prime}(K)$ is the group of all the roots of 1 the orders of which are prime to $p$. It is clear that $K^{\Delta_{g}}$ is contained in $\mu_{p}^{\prime}(K) \times\left(K^{\Delta_{g}}\right)^{(p)}$. Therefore $V$ satisfies the three conditions (i) (iii) in the previous section. Furthermore we have

$$
K^{\times} / V \cdot \mathrm{N}_{K / k}^{-1}(1)^{(p)} \cong X / \mathrm{N}_{K / k}^{-1}(1)^{(p)} \cong \mu_{p}(k) \cap \mathrm{N}_{K / k}\left(K^{\times}\right) .
$$

Therefore the abelian extension $L$ of $K$ corresponding to $V$ assures Theorem 2. The proof is completed.

Corollary 1. Suppose that $\mathfrak{S}^{(p)}(K / k) \neq\{1\}$, and put $q=\mid \mu_{p}(k) \cap$ $\mathrm{N}_{K / k}\left(K^{\times}\right) \mid$. There exists an abundant central p-extension of $K / k$ with the trivial genus field if and only if there exists an element $x$ of $U_{K}^{(p)}$ such that $\left\langle\mathrm{N}_{K / k}(x)\right\rangle=\mu_{p}(k) \cap \mathrm{N}_{K / k}\left(K^{\times}\right)$and $x^{q} \in K^{\Delta_{g}}$.

Proof. Suppose that there exists an $x$ of Corollary. Let $V$ be the group in the above proof of the theorem. Then it is easy to see that the abelian extension of $K$ corresponding to $\langle x\rangle \cdot V$ is a desired central 
extension of $K / k$. Conversely suppose that there exists an abundant central $p$-extension of $K / k$ with the trivial genus field. Let $W$ be the open subgroup of $K^{\times}$corresponding to it. Then we have $K^{\times}=W \cdot \mathrm{N}_{K / k}^{-1}(1)^{(p)}$ and $W \cap \mathrm{N}_{K / k}^{-1}(1)^{(p)}=\left(K^{\Delta_{9}}\right)^{(p)}$. Let $z$ be an element of $U_{K}^{(p)}$ such that $\left\langle\mathrm{N}_{K / k}(z)\right\rangle=\mu_{p}(k) \cap \mathrm{N}_{K / k}\left(K^{\times}\right)$. Then there are $x \in W$ and $y \in \mathrm{N}_{K / k}^{-1}(1)^{(p)}$ such that $z=x \cdot y$. Then $\mathrm{N}_{K / k}(x)=\mathrm{N}_{K / k}(z)$. Furthermore $x^{q}=z^{q} \cdot y^{-q} \in W \cap$ $\mathrm{N}_{K / k}^{-1}(1)^{(p)}=\left(K^{\lrcorner g}\right)^{(p)}$.

CoRollary 2. Suppose that there exist no abundant central p-extensions of $K / k$ with the trivial genus field. Then we have

$$
\left[L^{*}: K\right] \geq q / \max \left\{r=p^{n} \mid X^{q} \subset \mathrm{N}_{K / k}^{-1}(1)^{r} \cdot K^{\lrcorner^{\natural}}\right\} \geq p
$$

for every abundant central p-extension $L$ of $K / k$.

Proof. Put $W=\mathrm{N}_{L / K}\left(L^{\times}\right)$. Then $\left[L^{*}: K\right]=\left[K^{\times}: W \cdot \mathrm{N}_{K / k}^{-1}(1)\right]=\left[\mathrm{N}_{K / k}\left(K^{\times}\right)\right.$: $\left.\mathrm{N}_{K / k}(W)\right] \geq\left[\mu_{p}(k) \cap \mathrm{N}_{K / k}\left(K^{\times}\right): \mu_{p}(k) \cap \mathrm{N}_{K / k}(W)\right]=q / r$ with $r=\left|\mu_{p}(k) \cap \mathrm{N}_{K / k}(W)\right|$. Since $\mathrm{W} \cap \mathrm{N}_{K / k}^{-1}(1)^{(p)} \subset K^{\iota_{9}}$ by the choice of $L$, we easily see that $X^{q} \subset$ $\mathrm{N}_{K / k}^{-1}(1)^{r} \cdot K^{\lrcorner_{g}}$. It is also easy to see that the last condition of Corollary 1 holds if we have $X^{q} \subset \mathrm{N}_{K / k}^{-1}(1)^{q} \cdot K^{\Delta_{9}}$, which is not the case. Q.E.D.

In the rest of this section, let $k$ be a finite algebric extension of $\boldsymbol{Q}_{p}$ such that $q=\left|\mu_{p}(k)\right| \geq p$. Let $\zeta$ be a generator of $\mu_{p}(k)$.

ExAmPLE 2. Let $k_{1}$ be the unramified extension of $k$ of degree $q$, and $k_{2}$ be a totally ramified Kummer extension $k(\sqrt[q]{u})$ with $u \in U_{k}-k^{\times p}$ such that $\mu_{p}(k)=\langle\zeta\rangle \subset \mathrm{N}_{k_{2} / k}\left(k_{2}^{\times}\right)$. Put $K=k_{1} \cdot k_{2}$. Then Gal $(K / k)$ is abelian of type $(q, q)$. We show that there exists an abundant central $p$-extension of $K / k$ with the trivial genus field.

Take $x \in K^{\times}$satisfying $\mathrm{N}_{K / k}(x)=\zeta$. By Corollary 1 above, it is sufficient to show that

$$
\langle x\rangle \cap \mathrm{N}_{K / k}^{-1}(1)=\left\langle x^{q}\right\rangle \subset K^{\lrcorner g} .
$$

It is obvious that $\langle x\rangle \cap \mathrm{N}_{K / k}^{-1}(1)=\left\langle x^{q}\right\rangle$. Put $y=\mathrm{N}_{K / k_{1}}(x)$. Then $y \equiv x^{q}$ $\bmod K^{\Delta_{g}}$ since $\left[K: k_{1}\right]=q$. Let us show that $y$ belongs to $K^{\Delta_{9}}$. We have $\mathrm{N}_{K / k_{2}}(y)=\mathrm{N}_{k_{1} / k}(y)=\mathrm{N}_{K / k}(x)=\zeta$. Since $K / k_{2}$ is unramified, $\sqrt[q]{u}$ belongs to $\mathrm{N}_{K / k_{2}}\left(K^{\times}\right)$. Therefore $\zeta=(\sqrt[q]{u})^{1-\sigma} \in \mathrm{N}_{K / k_{2}}\left(K^{\times}\right)^{d_{g}}$, where $\sigma$ is a generator of $\operatorname{Gal}\left(k_{2} / k\right)$. Then we have the desired result by the following lemma.

Lemma 5. Let $K / k$ be a finite Galois extension of local fields, and $\mathrm{g}=\mathrm{Gal}(K / k)$. Let $M$ be an intermediate field of $K / k$, and suppose that 
$K / M$ is cyclic and that $M / k$ is normal. Then for $a \in K^{\times}$to belong to $K^{\Delta_{\mathrm{g}}}$, it is necessary and sufficient that $N_{K / M}(a) \in \mathrm{N}_{K / M}\left(K^{\times}\right)^{\Delta_{9}}$.

Proof. Since $\operatorname{Gal}(K / M)$ is a normal subgroup of $\mathrm{Gal}(K / k)$, we have $\mathrm{N}_{K / M}\left(K^{\Delta_{8}}\right)=\mathrm{N}_{K / M}\left(K^{\times}\right)^{\Delta_{\mathfrak{g}}}$. Suppose that $\mathrm{N}_{K / M}(a) \in \mathrm{N}_{K / M}\left(K^{\times}\right)^{\Delta_{\mathfrak{g}}}$. Take $x \in K^{\Delta_{8}}$ such that $\mathrm{N}_{K / M}(a)=\mathrm{N}_{K / M}(x)$. Then $\mathrm{N}_{K / M}\left(a x^{-1}\right)=1$. Therefore we can find $y \in K^{\times}$such that $a x^{-1}=y^{1-\sigma}$ where $\langle\sigma\rangle=\operatorname{Gal}(K / M)$. Thus we have $a=x \cdot y^{1-\sigma} \in K^{\lrcorner_{\mathfrak{g}}}$.

Q.E.D.

ExAmple 3. Let $k_{3}=k(\sqrt[q]{v})$ be a Kummer extension of $k$ such that (1) $v \in k^{\times}-k^{\times p}$, (2) $\left\langle v \bmod k^{\times q}\right\rangle \cap\left\langle\zeta \bmod k^{\times q}\right\rangle=\{1\}$ in $k^{\times} / k^{\times q}$, and (3) $\mathrm{N}_{k_{3} / k}\left(k_{3}^{\times}\right) \ni \zeta$. Let $k_{4}$ be a cyclic extension of degree $q$ over $k$ such that $\langle v\rangle \cdot \mathrm{N}_{k_{4} / k}\left(k_{4}^{\times}\right)=k^{\times}$and that $\mathrm{N}_{k_{4} / k}\left(k_{4}^{\times}\right) \ni \zeta$. If $k_{3}$ is chosen, then such $k_{4}$ exists. Put $K=k_{3} \cdot k_{4}$ this time. Then there is no abundant central $p$-extension of $K / k$ with the trivial genus field.

To see this, first observe that

$$
\mathrm{N}_{K / k_{3}}\left(K^{\times}\right)=\mathrm{N}_{k_{3} / k}^{-1}\left(\mathrm{~N}_{k_{4} / k}\left(k_{4}^{\times}\right)\right)=\mathrm{N}_{k_{3} / k}^{-1}\left(\mathrm{~N}_{k_{3} / k}\left(k_{3}^{\times}\right) \cap \mathrm{N}_{k_{4} / k}\left(k_{4}^{\times}\right)\right) .
$$

By the choice of $k_{4}$, therefore, we see that

$$
(\sqrt[q]{v})^{r} \cdot k^{\times} \cap \mathrm{N}_{K / k_{3}}\left(K^{\times}\right)=\phi
$$

for $r$, a power of $p$ less than $q$.

Let $x$ be an element of $K$ such that $\mathrm{N}_{K / k}(x)=\zeta$. Let us show that $\langle x\rangle \cap K^{\Delta_{\mathfrak{g}}}=\left\langle x^{q^{2}}\right\rangle$. Put $y=\mathrm{N}_{K / k_{4}}(x)$. It is sufficient to show that $y^{r} \in K^{\Delta_{\mathfrak{g}}}$ for $r=p^{t}$ implies $r \geq q$ since $y \equiv x^{q} \bmod K^{\Delta_{g}}$. We have $\mathrm{N}_{K / k_{3}}(y)=\zeta$. By the above lemma applied to $M=k_{3}$, it is enough to see that $\zeta^{r} \notin \mathrm{N}_{K / k_{3}}\left(K^{\times}\right)^{\Delta_{g}}$ if $r<q$. Let $\sigma$ be a generator of $\operatorname{Gal}\left(k_{3} / k\right)$ such that $(\sqrt[q]{v})^{\sigma-1}=\zeta$. We have

$$
\mathrm{N}_{K / k_{3}}\left(K^{\times}\right)^{\lrcorner_{\mathcal{g}}}=\left\{z^{\sigma-1} \mid z \in \mathrm{N}_{K / k_{3}}\left(K^{\times}\right)\right\} .
$$

Therefore if $\zeta^{r} \in \mathrm{N}_{K / k_{3}}\left(K^{\times}\right)^{\Delta_{9}}$, then $\zeta^{r}=z^{\sigma-1}$ for some $z \in \mathrm{N}_{K / k_{3}}\left(K^{\times}\right)$, and $\left\{z^{-1} \cdot(\sqrt[q]{v})^{r}\right\}^{\sigma-1}=1$. But then we have $z \in(\sqrt[q]{v})^{r} \cdot k^{\times}$, which contradicts $(* *)$ if $r<q$.

Remark. By a careful examination of Theorems 1 and 2 and their proofs, one can easily see the following.

Theorem 3. Let $K / k$ be a finite Galois extension of local fields of characteristic 0. Then for each prime $p$, there exists a Galois extension $K_{1}$ of $k$ which satisfies the following conditions: 
(1) $K_{1} \subset K \cdot k_{\mathrm{ab}}$;

(2) $K_{1} / K$ is cyclic of degree at most $\left|\mu_{p}(k) \cap \mathrm{N}_{K / k}\left(K^{\times}\right)\right|$;

(3) There exists an abundant central p-extension of $K_{1} / k$ with the trivial genus field $K_{1}$.

\section{§4. Global Results}

In order to apply our results to global fields, it is necessary to study the connection between central extensions of local and global fields. Initially we deal with the case where the decomposition group $\mathfrak{g}_{\mathfrak{B}}$ of some prime divisor $\mathfrak{P}$ in $K$ is simply $g$. This is sufficient for the construction of a global extension whose abundant central extension cannot have trivial genus. The more general situation can be analysed via cohomology.

Proposition 2. Let $K / k$ be a finite Galois extension of algebraic number fields, and $L$ be a finite central extension of $K / k$. Let $S$ be an intermediate field of $L / k$ such that $S \cap K=k$. Then $S$ is abelian over $k$. Moreover the genus field $L^{*}=L \cap K \cdot k_{\mathrm{ab}}$ of $L$ over $K / k$ coincides with the genus field of $L$ over $K S / S$.

Proof. Put $\mathbb{S}=\operatorname{Gal}(L / k), \quad \mathfrak{S}=\operatorname{Gal}(L / S), \quad \mathfrak{A}=\operatorname{Gal}(L / K)$ and $\mathfrak{N}=$ $\operatorname{Gal}(L / K S)=\mathfrak{U} \cap \mathfrak{S}$. Then $\mathfrak{A}$ lies in the centre of $\mathbb{S}$ since $L$ is central. Moreover $\mathbb{S}=\mathfrak{A} \cdot \mathscr{F}$ since $K \cap S=k$. Thus the commutator $\mathbb{S}^{\prime}$ of $\mathbb{S}$ is simply $\mathfrak{S E}^{\prime}$. Clearly $\mathfrak{S C}$ is normal, and $\operatorname{Gal}(S / k)$ is isomorphic to $\mathbb{S} / \mathfrak{S C} \cong \mathfrak{A} / \mathfrak{R}$, hence abelian. The genus field $L^{*}$ of $L$ over $K / k$ is characterised by $\operatorname{Gal}\left(L / L^{*}\right)=\mathscr{S}^{\prime} \cap \mathfrak{A}=\mathfrak{S}^{\prime} \cap \mathfrak{U}=\mathfrak{S}^{\prime} \cap \mathfrak{R}$. Thus $L^{*}$ is also the genus field of $L$ over $K S / S$.

Q.E.D.

We may now consider localisation.

Proposition 3. Let $K / k$ and $L$ be as above. Let $\mathfrak{p}$ be a prime divisor of $k$, and $k_{\mathfrak{p}}$ be the completion of $k$. Let $\tilde{K}=K \cdot k_{\mathfrak{p}}$ and $\tilde{L}=L \cdot k_{\mathfrak{p}}$ be the corresponding local extensions. Suppose that $\left[\tilde{K}: k_{p}\right]=[K: k]$. Then $\tilde{L}$ is a central extension of $\tilde{K} / k_{\mathfrak{p}}$ such that

$$
\operatorname{Gal}\left(\tilde{L} / \tilde{L}^{*}\right) \cong \operatorname{Gal}\left(L / L^{*}\right) .
$$

Proof. Let $\mathfrak{P}$ be a prime divisor of $L$ over $\mathfrak{p}$ such that the completion $L_{\Re}$ coincides with $\tilde{L}$, and let $S$ be the decomposition field of $\mathfrak{P}$ in $L / k$. Since $\left[\tilde{K}: k_{\mathrm{p}}\right]=[K: k]$, we have $K \cap S=k$. $\operatorname{Gal}\left(\tilde{L} / k_{\mathrm{p}}\right)$ is natually isomorphic to $\operatorname{Gal}(L / S)$ so that by Proposition 2 we have a natural isomorphism of $\operatorname{Gal}\left(\tilde{L} / \tilde{L}^{*}\right)$ onto $\operatorname{Gal}\left(L / L^{*}\right)$.

Q.E.D. 
Corollary 1. Let the notation and the assumption be as in Proposition 3. Then $L$ is an abundant central extension of $K / k$ if and only if $\tilde{L}$ is one for $\tilde{K} / k_{\mathrm{p}}$.

Proof. Since $\left[\tilde{K}: k_{p}\right]=[K: k]$, we have a natural isomorphism of $\operatorname{Gal}\left(\tilde{K} / k_{\mathfrak{p}}\right)$ onto $\mathrm{Gal}(K / k)$, which induces an isomorphism of $\mathrm{H}^{2}\left(\mathrm{Gal}\left(\tilde{K} / k_{\mathfrak{p}}\right)\right.$, $\boldsymbol{Q} / Z)$ onto $\mathrm{H}^{2}(\mathrm{Gal}(K / k), \boldsymbol{Q} / Z)$. The corollary is then immediate from Proposition 3 .

Corollary 2. Let the notation and the assumption be as in Proposition 3. Let $S$ be the decomposition field of a prime divisor of $L$ dividing $\mathfrak{p}$. Then we have

$$
\left[L^{*}: K\right]=\left[\tilde{L}^{*}: \tilde{K}\right] \cdot[S: k] .
$$

Proof. We have $K \cap S=k$. Therefore $K S$ is contained in $L^{*}$ by Proposition 2. Thus we have $\left[L^{*}: K\right]=\left[L^{*}: K S\right] \times[K S: K]$. However $[K S: K]=[S: k]$. Furthermore it follows from Proposition 3 that

$$
\left[\tilde{L^{*}}: \tilde{K}\right]=[\tilde{L}: \tilde{K}] /\left[\tilde{L}: \tilde{L}^{*}\right]=[L: K S] /\left[L: L^{*}\right]=\left[L^{*}: K S\right] .
$$

Therefore we have $\left[L^{*}: K\right]=\left[\tilde{L}^{*}: \tilde{K}\right] \cdot[S: k]$.

Q.E.D.

More generally, let $\mathfrak{g}=\operatorname{Gal}(K / k)$ and $\mathfrak{g}_{\mathfrak{p}}=\operatorname{Gal}\left(\tilde{K} / k_{\mathfrak{p}}\right)$. Then we have a commutative diagram

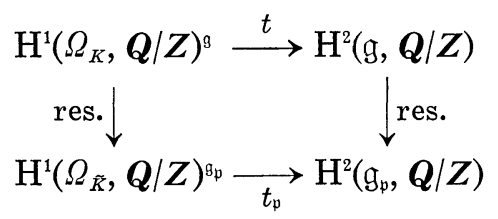

where the vertical maps are restriction and the horizontal maps are transgression. It is clear that Proposition 3 remains true so long as

$$
\text { Res: } \mathrm{H}^{2}(\mathrm{~g}, \boldsymbol{Q} / \boldsymbol{Z}) \longrightarrow \mathrm{H}^{2}\left(\mathfrak{g}_{\mathfrak{p}}, \boldsymbol{Q} / Z\right)
$$

is injective.

Similarly an abundant central extension of a Galois]? global fields remains abundant upon localisation so long as

$$
\text { Res: } \mathrm{H}^{2}(\mathfrak{g}, \boldsymbol{Q} / Z) \longrightarrow \mathrm{H}^{2}\left(\mathfrak{g}_{p}, \boldsymbol{Q} / Z\right)
$$

is surjective.

Thus Corollary 1 remains true if the condition $\left[\tilde{K}: k_{p}\right]=[K: k],{ }^{7}$ i.e. $\mathrm{g} \cong \mathfrak{g}_{\mathfrak{p}}$, is replaced by the weaker condition that 


$$
\text { Res: } \mathrm{H}^{2}(\mathfrak{g}, \boldsymbol{Q} / Z) \longrightarrow \mathrm{H}^{2}\left(\mathfrak{g}_{*}, \boldsymbol{Q} / Z\right)
$$

is an isomorphism.

Example 4. Now we give an example of a global extension $K / \boldsymbol{Q}$ any abundant central extension of which cannot have the trivial genus field.

Fix a prime $p$ and an integer $m \geq 2$. Let $\ell$ be a prime number such that $p^{m} \| \ell-1$. Then the $\ell$-adic completion $\boldsymbol{Q}_{\ell}$ contains a primitive $p^{m}$-th root $\zeta_{m}$ of 1 but does not $\zeta_{m+1}$. The group of $p$-power roots of 1 in $\boldsymbol{Q}_{\ell}\left(\zeta_{m+1}\right)$ is just $\left\langle\zeta_{m+1}\right\rangle$.

Let $k_{1}$ and $k_{2}$ be cyclic extensions of $\boldsymbol{Q}$ of degree $p$ such that $\ell$ is ramified in $k_{1} / \boldsymbol{Q}$ but is unramified and remains prime in $k_{2} / \boldsymbol{Q}$. Put $K=k_{1} \cdot k_{2}$. Then $\operatorname{Gal}(K / Q)$ is abelian of type $(p, p)$. Therefore $\subseteq(K / Q)=$ $\widetilde{S}^{(p)}(K / \boldsymbol{Q})$ is a cyclic group of order $p$.

TheOREM 4. Let $L$ be an abundant central extension of $K / Q$. Then $\left[L^{*}: K\right] \geq p^{m-1} \geq p$. Therefore there is no abundant central extensions of $K / \mathbf{Q}$ with the trivial genus field.

Proof. Let $\tilde{K}$ be the local extension $K \cdot \boldsymbol{Q}_{\ell}$ of $\boldsymbol{Q}_{\ell}$. Then $\operatorname{Gal}\left(\tilde{K} / \boldsymbol{Q}_{\ell}\right)$ is naturally isomorphic to $\operatorname{Gal}(K / \boldsymbol{Q})$. Therefore $\mathfrak{S}^{(p)}\left(\tilde{K} / \boldsymbol{Q}_{\ell}\right) \cong \mathfrak{S}(K / \boldsymbol{Q})$ is not trivial. Since $\mu_{p}(\tilde{K})=\left\langle\zeta_{m+1}\right\rangle$, we have

$$
\left|\mathrm{N}_{\tilde{K} / Q_{\ell}}\left(\mu_{p}(\tilde{K})\right)\right|=\left|\left\langle\zeta_{m-1}\right\rangle\right|=p^{m-1} \geq p .
$$

The theorem is now an immediate consequence from Corollaries 1 and 2 above and Theorem 1 in Section 2.

\section{REFERENCES}

[1] A. Fröhlich, On fields of class two, Proc. London Math. Soc. (3), 4 (1954), 235-256.

[ 2 ] - (ed.), Algebraic Number Fields, Acad. Press, London: New York: San Francisco, 1977.

[ 3 ] G. Hochschild and J.-P. Serre, Cohomology of group extensions, Trans. Amer. Math. Soc., 74 (1953), 110-134.

[4] K. Miyake, Central extensions and Schur's multiplicators of Galois groups, Nagoya Math. J., 90 (1983), 137-144.

[5] — - On central extensions of a Galois extension of algebraic number fields, Nagoya Math. J., 93 (1984), 133-148.

[6 ] J.-P. Serre, Modular forms of weight one and Galois representations, in Fröhlich [2], 193-268. 
K. Miyake

Department of Mathematics

College of General Education

Nagoya University

Nagoya 464, Japan

N. Ormerod

School of Mathematics

The University of New South Wales

PO Box 1, Kensington

N.S.W. 2033, Australia 